\title{
Preparation and preliminary evaluation of hepatitis B core antigen virus like nanoparticles loaded with indocyanine green
}

\author{
Yunlong Wang ${ }^{1,2}$, Yiqing Zhang ${ }^{1,2}$, Yinyin Yu ${ }^{3}$, Lei Ren ${ }^{4}$, Jichuang Wang ${ }^{1}$, Lei Cheng ${ }^{1}$, Dandan Jiang ${ }^{1}$, \\ Xiangqian Guo ${ }^{5}$, Tieshan Teng ${ }^{5}$, Xiaoyong Luo $^{2}$, Shuangyu $\mathbf{L v}^{5}$, Xudong Wang ${ }^{3}$, Huirui Wang ${ }^{6}$, \\ Xinpeng Shi ${ }^{6}$, Heng Zhang ${ }^{3}$, Shengli $\mathrm{Bi}^{1}$
}

${ }^{1}$ Henan Bioengineering Research Center, Zhengzhou, China; ${ }^{2}$ Zhengzhou Technical College, Zhengzhou, China; ${ }^{3}$ Henan General Hospital, Zhengzhou, China; ${ }^{4}$ Department of Biomaterials, Key Laboratory of Biomedical Engineering of Fujian Province, State Key Laboratory of Physical Chemistry of Solid Surface, College of Materials, Xiamen University, Xiamen, China; ${ }^{5}$ School of Basic Medical Sciences, Henan University, Kaifeng, China; ${ }^{6}$ Department of Radiation Oncology, Luoyang Central Hospital Affiliated to Zhengzhou University, Luoyang, China

Contributions: (I) Conception and design: Y Wang, S Bi, X Luo; (II) Administrative support: Y Wang; (III) Provision of study materials or patients: Y Wang, S Bi, X Luo; (IV) Collection and assembly of data: S Bi, Y Wang, X Luo, S Lv; (V) Data analyses and interpretation: X Luo, S Bi, S Lv; (VI) Manuscript writing: All authors; (VII) Final approval of manuscript: All authors.

Correspondence to: Dr. Shengli Bi. Henan Bioengineering Research Center, Zhengzhou 450046, China. Email: bisl@ivdc.chinacdc.cn; Dr. Yunlong Wang. Henan Bioengineering Research Center, Zhengzhou 450046, China. Email: biowyl@126.com; Dr. Xiaoyong Luo. Department of Radiation Oncology, Luoyang Central Hospital Affiliated to Zhengzhou University, Luoyang 471000, China. Email: zlklxy68@163.com; Dr. Shuangyu Lv. School of Basic Medical Sciences, Henan University, Kaifeng 475004, China. Email: shuangyulv@henu.edu.cn.

Background: In recent years, nanotechnology has attracted a plethora of attention due of its ability to effectively diagnose and treat various tumors. Virus-like particles (VLPs) have good biocompatibility, are safe and non-toxic, and have an internal hollow space, and as such they are often used as nano drug carriers. In recent years, it has become one of the hot spots in the field of biopharmaceutical engineering.

Methods: In this study, the tumor-targeting peptide RGD (Arg-Gly-Asp) was genetically inserted into the major immunodominant region (MIR) of the hepatitis B virus core protein ( $\mathrm{HBc}$ ). A series of characterization, including stability and optical properties, were evaluated. A visual diagnosis and analysis of the efficacy against tumor cells were conducted at the cell level and using a live animal model.

Results: This study demonstrated that the recombinant HBc-based VLPs could participate in self-assembly of monodispersed nanoparticles with well-defined morphology, and the near-infrared dye indocyanine green (ICG) could be packaged into the VLPs without any chemical modification. Moreover, the HBc-based VLPs could specifically target cancer cells via the interaction with overexpressed integrin $\alpha v \beta 3$. The treatment with ICG-loaded HBc-based VLPs showed significant inhibition of 4T1 breast cancer cell growth (84.87\% tumor growth inhibition). The in vivo imaging experiments demonstrated that the ICG-loaded HBc-based VLPs generated excellent fluorescence in tumor sites in 4T1 breast cancer bearing mice. This provided crucial information on tumor mass location, boundaries, and shape. Moreover, compared to free ICG, the nanosystem showed significantly longer blood circulation time and superior accuracy in targeting the tumor.

Conclusions: The ICG-loaded HBc-based VLPs prepared in this study were of good stability and biocompatibility. It showed strong tumor targeting specificity and tumor visualization. Thus, it is expected to provide a new experimental basis and theoretical support for the integration of VLPs in the clinical diagnosis and treatment of breast cancer.

Keywords: Hepatitis B core protein (HBc); virus-like particles (VLPs); drug delivery; tumor targeting; breast cancer

Submitted Oct 23, 2020. Accepted for publication Dec 16, 2020.

doi: $10.21037 / \mathrm{atm}-20-7478$

View this article at: http://dx.doi.org/10.21037/atm-20-7478 


\section{Introduction}

In the field of cancer, nanotechnology has increasingly been applied to the screening, diagnosis, and treatment of malignancies. Nanomedicine involves the nanoscale application of highly specific medical interventions. Compared with conventional cancer interventions, nanomedicine has unique features, such as the ability to specifically detect and target tumors $(1,2)$. In cancer treatment, nanomedicine not only improves the therapeutic index of traditional drugs, but also provides innovative ideas for novel treatment modalities (3). These attractive advantages have inspired scientists to pursue research in cancer nanomedicine, contributing to the development of promising treatments to fight cancer in the future. To date, nanotechnology has been applied in the detection and diagnosis of various cancers, such as breast cancer, lung cancer, gastric cancer, etc. (4).

The hepatitis $\mathrm{B}$ virus core protein $(\mathrm{HBc})$ was first described as virus-like particles (VLPs). It is composed of 183-188 amino acids with a molecular weight of approximately $21 \mathrm{kDa}$ and has a positive icosahedral particle structure. The first 1-144 amino acids represent the assembly region and are necessary for the construction of the particles $(5,6)$. In fact, it has been shown that truncated HBc (1-144 aa, HBc-144) expressed by Escherichia coli could self-assemble into VLPs that were similar to the wild type in morphology and physical properties (7). Among them, 72-82 amino acids present a surface spike-like structure as seen under the electron microscope, while 79 amino acids are located at the top of the spike, which are the main immunogenic regions of $\mathrm{HBc}$ and can be used as targets for gene or chemical modification $(8,9)$. The HBC has a clear structure, good self-assembly, good biocompatibility, safety and non-toxic, and has a hollow inner space, which can be used as a nano drug carrier. To date, HBc VLPs have shown great promise as viral vaccines and tumor vaccines. Duan and colleagues (10) fused epidermal growth factor type III mutants on the surface of $\mathrm{HBc}$ VLPs and immunized mice with the fused product. The tumor that was subsequently transplanted showed significant necrosis, which suggested that the fused HBc VLPs could significantly inhibit the growth of the transplanted tumor in the mice. The CpG (cytosine linked to a guanine by a phosphate bond) oligodeoxynucleotide consists of a series of cytosineguanine motifs and can act as an immunostimulants (11). Dendritic cells stimulated by HBc VLPs coated with CpG oligodeoxynucleotides could induce a strong $\mathrm{T}$ helper cell and cytotoxic T lymphocyte (CTL) response, thereby inhibiting the growth of tumors in mice, and it was indicated that HBc VLPs packaging CpG-pulsed dendritic cells could facilitate the development of effective T-cellbased vaccines (12). In addition, Shan et al. (13) loaded the chemotherapy drug doxorubicin (DOX) into HBc VLPs. In the B16F10 tumor-bearing mouse model, DOX-loaded VLPs had a significant inhibitory effect on tumor growth [90.7\% tumor growth inhibition (TGI)], and a markedly lower cardiotoxic effect than animals treated with DOX alone.

At present, many nanomaterials, such as polymer nanoparticles, liposomes, gold nanoparticles and hydrogels, are studied for the diagnosis of breast cancer. Comparing with other nano carriers, the HBc/ICG VLPs have the following advantages. First, HBc/ICG VLPs have more clear structure and better particle uniformity. Second, there are many binding sites on the surface of HBc VLPs which can be modified and easy to add targeted peptides and charged amino acids to improve the targeting and drug loading. Third, HBc VLPs have good biological safety. They can be quickly metabolized and decomposed by enzymes in tumor site, and have no side effects (14-16). Additionally, several nano carriers containing indocyanine green (ICG) are used for photothermal therapy $(17,18)$.

We prepared a new type of $\mathrm{HBc}$ virus-like nanoparticle modified with the tumor-targeting peptide RGD (consisting of Arginine-Glycine-Aspartate). The RGD-HBc VLPs were prepared through depolymerization and self-assembly under specific conditions in vitro, and the near-infrared dye ICG was loaded into them. Examination of the resultant RGD-HBc/ICG VLPs demonstrated that the stability and targeting specificity of ICG were improved, the enrichment and retention time of ICG in tumor sites were prolonged, and the tumor diagnostic imaging was effectively improved. We present the following article in accordance with the ARRIVE reporting checklist (available at http://dx.doi. org/10.21037/atm-20-7478).

\section{Methods}

\section{Preparation of the RGD-HBc VLPs nanoparticles}

The RGD-HBc VLPs were prepared in our laboratory. The core antigen $1-144$ of the hepatitis B virus was obtained from the National Center for Biotechnology Information (NCBI). The RGD (Arg-Gly-Asp) sequence was inserted into the major immunodominant region (MIR) of the HBc 
VLPs thereby allowing recognition of tumor cell integrins. The gene sequence was synthesized by a biological company (Sangon Biotech Co., Ltd., Shanghai, China) and inserted into plasmids. The plasmids were then transformed into receptive cells and the recombinant protein was induced and expressed. Finally, the RGD-HBc VLPs were collected by chromatography and sucrose density gradient centrifugation.

\section{Transmission electron microscopy (TEM) and Malvern particle size characterization}

The purity of the RGD-HBc VLPs were determined using the Quantity One analysis of the Bio-Rad gel imaging system (Gel DocTM XR ${ }^{+}$). The samples of RGD-HBc VLPs purified by molecular sieve chromatography, anionexchange chromatography, and ultracentrifugation in sucrose gradients were detected by sodium dodecylsulphate polyacrylamide gel electrophoresis (SDS-PAGE) electrophoresis. The morphology and size of the RGD-HBc VLPs were characterized by TEM. Samples of the RGD$\mathrm{HBc}$ VLPs were diluted to $0.5 \mathrm{mg} / \mathrm{mL}$, and an aliquot was dropped onto a copper net. Samples were then negative dyed with $5 \%$ uranium acetate for 5 minutes, dried at room temperature, and observed under TEM. The morphology and size of the RGD-HBc VLP nanoparticles were examined to determine whether the particles were complete and uniform. The particle size of the nanoparticles were analyzed by a MasterSizer Laser Diffraction Particle size Analyzer (Wano-2s, Malvern Instruments Ltd., Malvern, England). Nanoparticles were dilute to $0.5 \mathrm{mg} / \mathrm{mL}$ and placed in the particle size cup and the potential cup of the Malvern detector for detection. Each sample was analyzed in triplicates.

\section{RGD-HBc VLPs physical stability test}

To explore the long-term stability of the RGD-HBc VLPs following prolonged storage at $4{ }^{\circ} \mathrm{C}$, the above-mentioned RGD-HBc VLP samples were purified by sucrose density gradient centrifugation and diluted to $0.5 \mathrm{mg} / \mathrm{mL}$. The RGD-HBc VLP samples were observed by TEM on the same day, or stored at $4{ }^{\circ} \mathrm{C}$ for 30 days prior to TEM examination. The VLPs were prepared and observed by TEM as detailed above. Changes in the size and morphology of the VLPs were analyzed.

The $\mathrm{pH}$ value is one of the factors that affect the stability of a protein. In a wide range of $\mathrm{pH}$ tests, five identical samples of ultracentrifuged RGD-HBc VLPs were resuspended in phosphate buffered saline (PBS) buffer with a $\mathrm{pH}$ of $5,6,7,8$, or 9. The purity of the RGD-HBc VLPs was determined by high performance liquid chromatography (HPLC) at $4{ }^{\circ} \mathrm{C}$. The HPLC machine was prepared as per manufacturer's instructions (e2695, Waters Technology, Milford, MA, USA). The samples were filtered with a $0.44 \mu \mathrm{m}$ filter and $20 \mu \mathrm{L}$ of sample was loaded into the column. The column temperature was set at $25^{\circ} \mathrm{C}$, and the mobile phase used was $20 \mathrm{~mm}$ PBS buffer and $0.15 \mathrm{M}$ sodium chloride ( $\mathrm{pH} 7.4$ ). The detection wavelength used was $280 \mathrm{~nm}$, the flow rate was $0.6 \mathrm{~mL} / \mathrm{min}$, and the elution time was 30 minutes.

\section{Preparation of the RGD-HBc/ICG VLPs with ICG}

The above purified RGD-HBc VLP solution $(10 \mathrm{~mL}$ at $0.5 \mathrm{mg} / \mathrm{mL}$ ) was incubated with the depolymerized buffer for 2 hours at $25^{\circ} \mathrm{C}$ in a water bath. During this period, the solution was inverted gently every 30 minutes until the buffer was fully in contact with the nanoparticles. Subsequently, $2.5 \mathrm{~mL}$ of ICG solution was added to the nanoparticles and incubated, with stirring, in an ice bath for 1 hour. The mixed solution was then put into a dialysis bag (retained molecular weight: $8 \mathrm{KDa}$ ), and stirred overnight in the recombination buffer solution at $4{ }^{\circ} \mathrm{C}$, away from light. The dialytic solution was replaced twice during this period. The unencapsulated free ICG was removed by sucrose density gradient centrifugation.

\section{Optical performance characterization}

The optical properties were measured by UV (ultraviolet visible) spectrophotometry. Following baseline scanning with PBS buffer, the RGD-HBc VLPs, ICG, and RGD$\mathrm{HBc} / \mathrm{ICG}$ VLPs were placed in cuvettes for full wavelength scanning.

\section{Photothermal transformation performance test}

The ICG and RGD-HBc/ICG VLP solutions were prepared with PBS buffer and samples were placed in centrifuge tubes and irradiated with a $808 \mathrm{~nm}$ laser $\left(1.5 \mathrm{~W} / \mathrm{cm}^{2}\right)$ adjusted to 0.5 and $1 \mathrm{~W}$, respectively. Samples $(10,20$, 30 or $40 \mu \mathrm{g} / \mathrm{mL}$ ) were irradiated for 5 minutes, and the temperature was recorded with an infrared thermal imaging camera every 1 minute. 


\section{Singlet oxygen detection}

Samples of the PBS solution, the ICG solution, and the RGD-HBc/ICG VLP solution were placed into 96 well plates. The 1,3-diphenylisobenzofuran (DPBF) solution in the singlet oxygen detection kit (Shanghai Beibo Biotechnology Ltd., Shanghai, China) was diluted to $1 \mu \mathrm{g} / \mathrm{mL}$ and added to the 96 well plate. All samples were then irradiated with an $808 \mathrm{~nm}$ laser $\left(1.5 \mathrm{~W} / \mathrm{cm}^{2}\right)$ for 5 minutes, and the ultraviolet absorbance of DPBF at $410 \mathrm{~nm}$ was detected every 5 minutes. Each set was repeated three times and the average values were calculated.

\section{In vitro cellular uptake}

The 4T1 cells in the logarithmic growth stage were prepared to a single cell suspension, and then they were inoculated in 96 well plates with $1 \times 10^{4} /$ well in a volume of $100 \mu \mathrm{L}$. After being placed in the carbon dioxide incubator for 12 hours, the cells were attached to the wall, and then was washed 3 times with PBS buffer for 3 times. The complete culture medium within ICG and RGD-HBc/ ICG VLP at a final concentration of $40 \mu \mathrm{g} / \mathrm{mL}$ were added respectively, and incubated for 4, 8, 12 and 24 hours. After rinse with PBS buffer for 3 times, $100 \mu \mathrm{L}$ DAPI solution $(1 \mu \mathrm{g} / \mathrm{mL})$ was added to stain for 20 minutes under dark condition. Then, discard and rinse off the DAPI solution with PBS buffer, the uptake of RGD-HBc/ICG VLP was immediately observed by fluorescence microscope.

\section{In vitro light kill experiment}

The 4T1 cells in logarithmic growth phase were prepared into single cell suspension, and the cells were placed in a 12 well plate with $1 \times 10^{5} /$ well in a volume of $100 \mu \mathrm{L}$. The old culture medium was discarded after the cells adhered to the wall, and then the cells were washed three times using PBS buffer. The DMEM containing ICG and RGD-HBc/ICG VLP were added at a final concentration of $0,10,20,30$ and $40 \mu \mathrm{g} / \mathrm{mL}$, respectively. After culture for 12 hours, the cells were rinsed with PBS buffer for 3 times. Subsequently, the fresh culture medium was added and they irradiated with $808 \mathrm{~nm}$ laser $\left(1.5 \mathrm{w} / \mathrm{cm}^{2}, 5 \mathrm{~min}\right)$. The cells treated with laser were placed in carbon dioxide incubator, incubating for 12 hours. After rinse it with PBS buffer for 3 times, the cells were incubated with $10 \mu \mathrm{L}$ CCK-8 solution and $90 \mu \mathrm{L}$ DMEM in carbon dioxide incubator for 2 hours. Then, they were placed in a 96 well plate and the absorbance was detected using enzyme labeled instrument at $450 \mathrm{~nm}, 3$ replicates per well. The cell survival rate was calculated.

\section{Near infrared fluorescence imaging in vivo}

The 4T1 mouse breast cancer model was established using $\mathrm{BALB} / \mathrm{c}$ female mice. When the tumor volume of mouse breast cancer bearing mice is about $100 \mathrm{~mm}^{3}$, the near infrared fluorescence in vivo imaging was carried out. Ten breast cancer bearing mice were randomly selected and randomly divided into two groups (5 mice per group), including ICG group and RGD-HBc/ICG VLP group. The ICG solution or RGD-HBc/ICG VLP solution was administered by tail vein injection using a $1 \mathrm{~mL}$ syringe in a volume of $200 \mu \mathrm{L}$. The content of ICG was $1.0 \mathrm{mg} /$ $\mathrm{kg}$. Four hours after injection, IVIS lumina III was used for the first near-infrared fluorescence imaging in vivo. Mice were anesthetized with fluothane before imaging, and they were given sufficient oxygen to ensure their normal survival state during the imaging process. The images were taken at 4,8 and $12 \mathrm{~h}$ after injection using a small animal in vivo fluorescence imager (PerkinElmer, Waltham, MA, USA) under the same parameters, and the fluorescence distribution at each time point was collected.

\section{Ethic}

The animal study was reviewed and approved by the Committee on Animal Care and Use of Henan Bioengineering Research Center (Zhengzhou, China). The animal experimental procedures were in accordance with the guidelines approved by the Committee on Animal Care and Use of Henan Bioengineering Research Center (Zhengzhou, China).

\section{Statistical analysis}

Values are expressed as means \pm standard error of the means (S.E.M.). Data for multiple comparisons was evaluated by one-way ANOVA followed by Dunnett's multiple comparison using SPSS 16.0. The unpaired $t$-test was applied to compare difference between the two groups. Statistical significance was determined at $\mathrm{P}<0.05$.

\section{Results}

\section{Characterization of the RGD-HBc VLPs}

Quantity One analysis showed that the purity of the RGD-HBc VLPs reached 92\% (Figure 1A). The lanes 


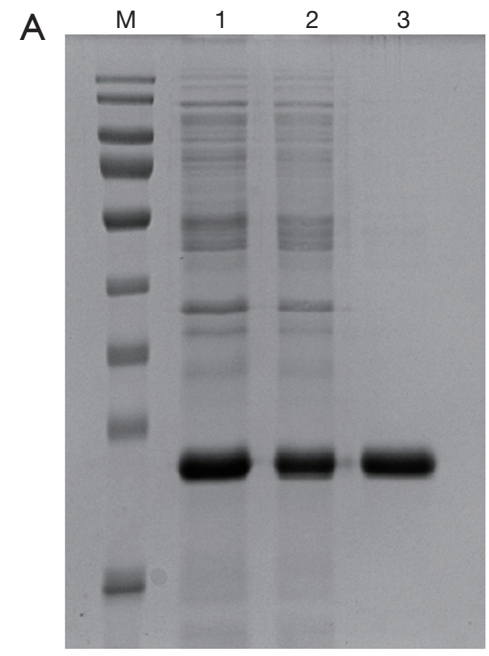

B
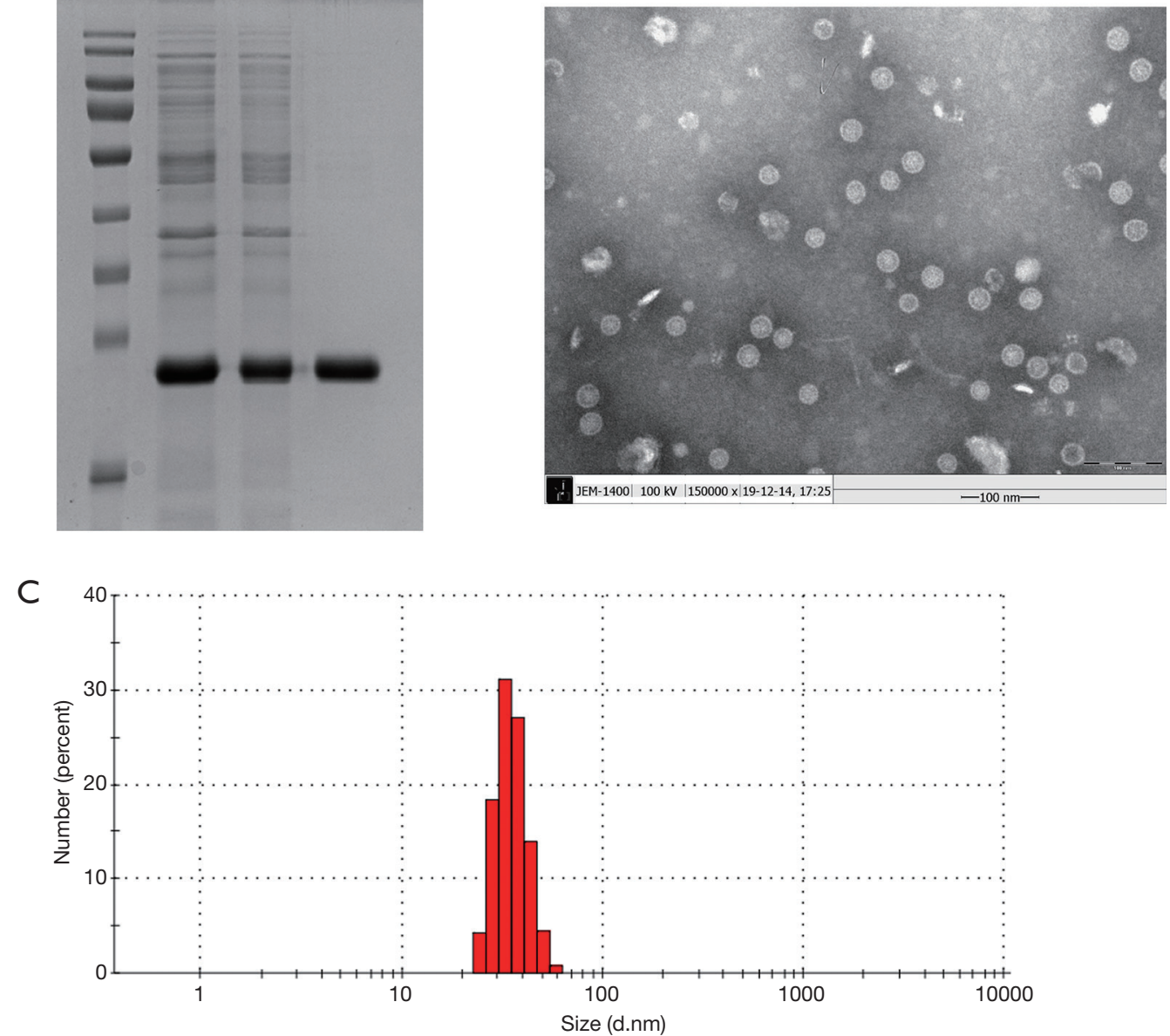

Figure 1 SDS-PAGE (A), TEM (B) and particle size (C) of the RGD-HBc VLPs. SDS-PAGE, sodium dodecylsulphate polyacrylamide gel electrophoresis; TEM, transmission electron microscopy; RGD, Arg-Gly-Asp; HBc, hepatitis B virus core protein; VLPs, virus-like particles; lane M, a protein marker; lane 1, molecular-sieve chromatography; lane 2, anion-exchange chromatography; lane 3, sucrose ultracentrifugation.

from 1 to 3 indicated the products purified by molecularsieve chromatography, anion-exchange chromatography, and sucrose ultracentrifugation, respectively. The lane $M$ indicated a protein marker. Under TEM, the morphology and size of the particles were uniform, and the diameter was about 28-32 nm (Figure 1B), which was consistent with the Malvern particle size detection results (Figure 1C).

\section{Stability of the RGD-HBc VLPs}

Stability is one of the important indexes to evaluate protein quality. The physical properties of the RGD-HBc VLPs, including the physical stability at different $\mathrm{pH}$ gradients, and following storage at $4{ }^{\circ} \mathrm{C}$ for 30 days, were examined.
The results of the TEM showed that storage of the RGD$\mathrm{HBc}$ VLPs at $4^{\circ} \mathrm{C}$ for 30 days did not affect the morphology and size compared to fresh RGD-HBc VLPs (Figure $2 A$ vs. Figure $1 B)$. The RGD-HBc VLPs were examined under different $\mathrm{pH}$ conditions by HPLC (Figure $2 B$ ). In the $\mathrm{pH}$ range of 7.0-8.0, except for the main peak, there were no large impurity peaks, which indicated that the RGD-HBc VLPs could be stored stably under these $\mathrm{pH}$ conditions.

\section{Characterization of the RGD-HBc/ICG VLPs}

The RGD-HBc VLPs were loaded with ICG. The TEM results demonstrated that the RGD-HBc/ICG VLPs were uniform in size and morphology (Figure $3 A$ ). The particle 

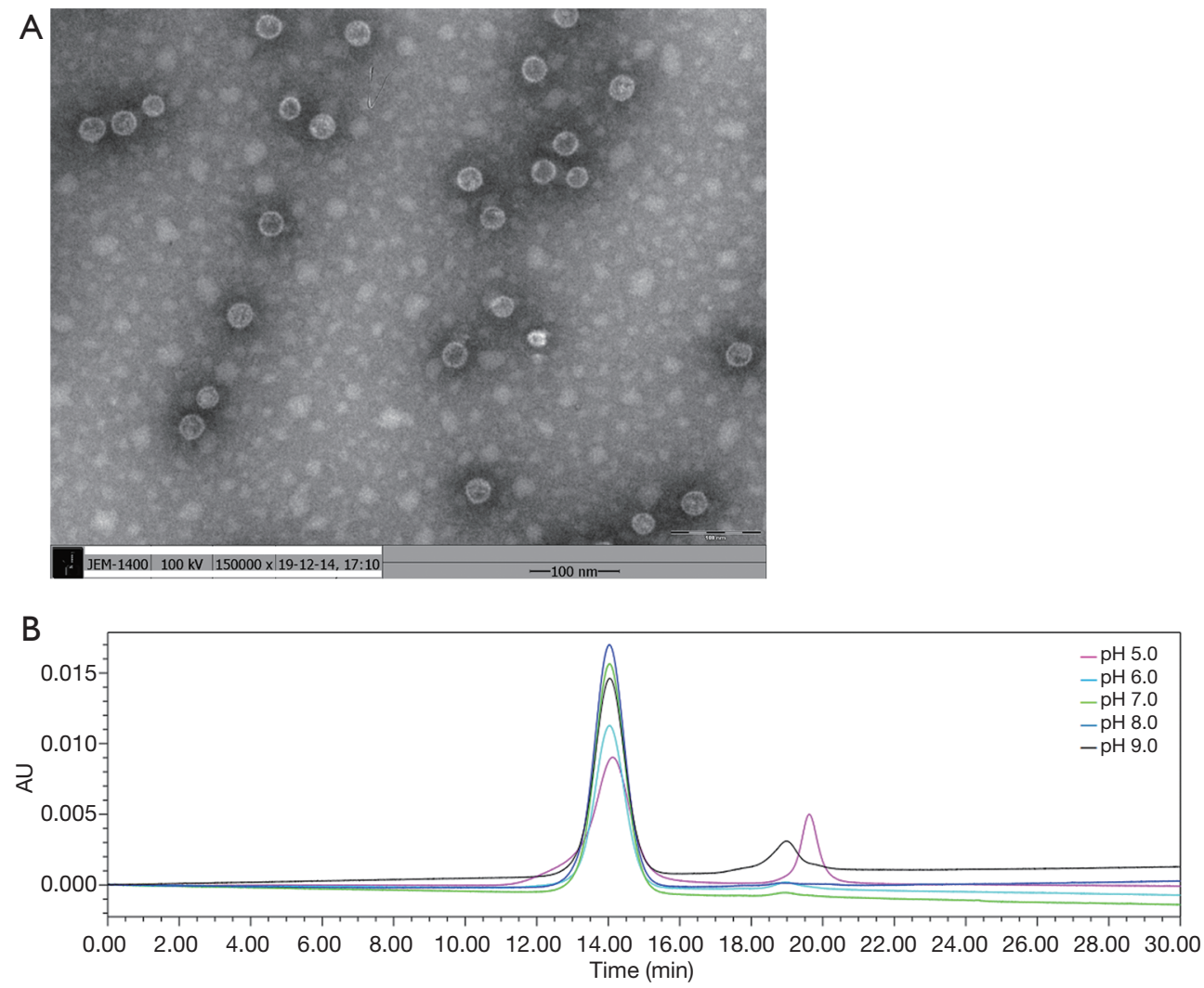

Figure 2 The stability test of the RGD-HBc VLPs. (A) Electron microscopy of the RGD-HBc VLPs following storage at $4{ }^{\circ} \mathrm{C}$ for 30 days; (B) The stability of the RGD-HBc VLPs at different $\mathrm{pH}$ determined by HPLC. RGD, Arg-Gly-Asp; HBc, hepatitis B virus core protein; VLPs, virus-like particles; HPLC, high performance liquid chromatography.

size was approximately $28-32 \mathrm{~nm}$ as shown in Figure $3 B$. Compared with the RGD-HBc VLPs (Figure 1), the morphology and size of the RGD-HBc/ICG VLPs did not changed significantly following ICG loading.

\section{Optical performance test}

Figure 4 shows the UV absorption of the full visible spectrum scan of ICG, the RGD-HBc VLPs and the RGD$\mathrm{HBc} / \mathrm{ICG}$ VLPs. The characteristic absorption peaks of ICG could be seen at 780 and $710 \mathrm{~nm}$. The RGD-HBc VLPs showed strong absorption at $280 \mathrm{~nm}$. After ICG loading, the RGD-HBc/ICG VLPs not only showed strong absorption at $280 \mathrm{~nm}$, but the strong ICG characteristic absorption peaks at 780 and $710 \mathrm{~nm}$ were also observed. This demonstrated that the RGD-HBc/ICG VLPs had been successfully prepared.

\section{Stability test}

As ICG is prone to degradation and photobleaching, the imaging quality and dynamic photothermal effects can be seriously affected. The physical and optical stability of ICG and the RGD-HBc/ICG VLPs were examined. From Figure $5 \mathrm{~A}$, it can be seen that after 10 days of storage at $4{ }^{\circ} \mathrm{C}$, the absorbance of ICG decreased by $77 \%$ (from 5.64 to 1.32). However, the absorbance of the RGD-HBc/ICG VLPs only decreased by $6 \%$, from 4.42 to 4.16 . The optical stability of ICG under $808 \mathrm{~nm}$ laser irradiation was also investigated. It can be seen from Figure $5 B$ that after 5 cycles of laser irradiation, the photothermal conversion effects of ICG worsened, while the photothermal conversion effects of the RGD-HBc/ICG VLPs were almost unchanged. These results indicated that the encapsulation of ICG in the RGD-HBc VLPs protected ICG from degradation and photobleaching. 

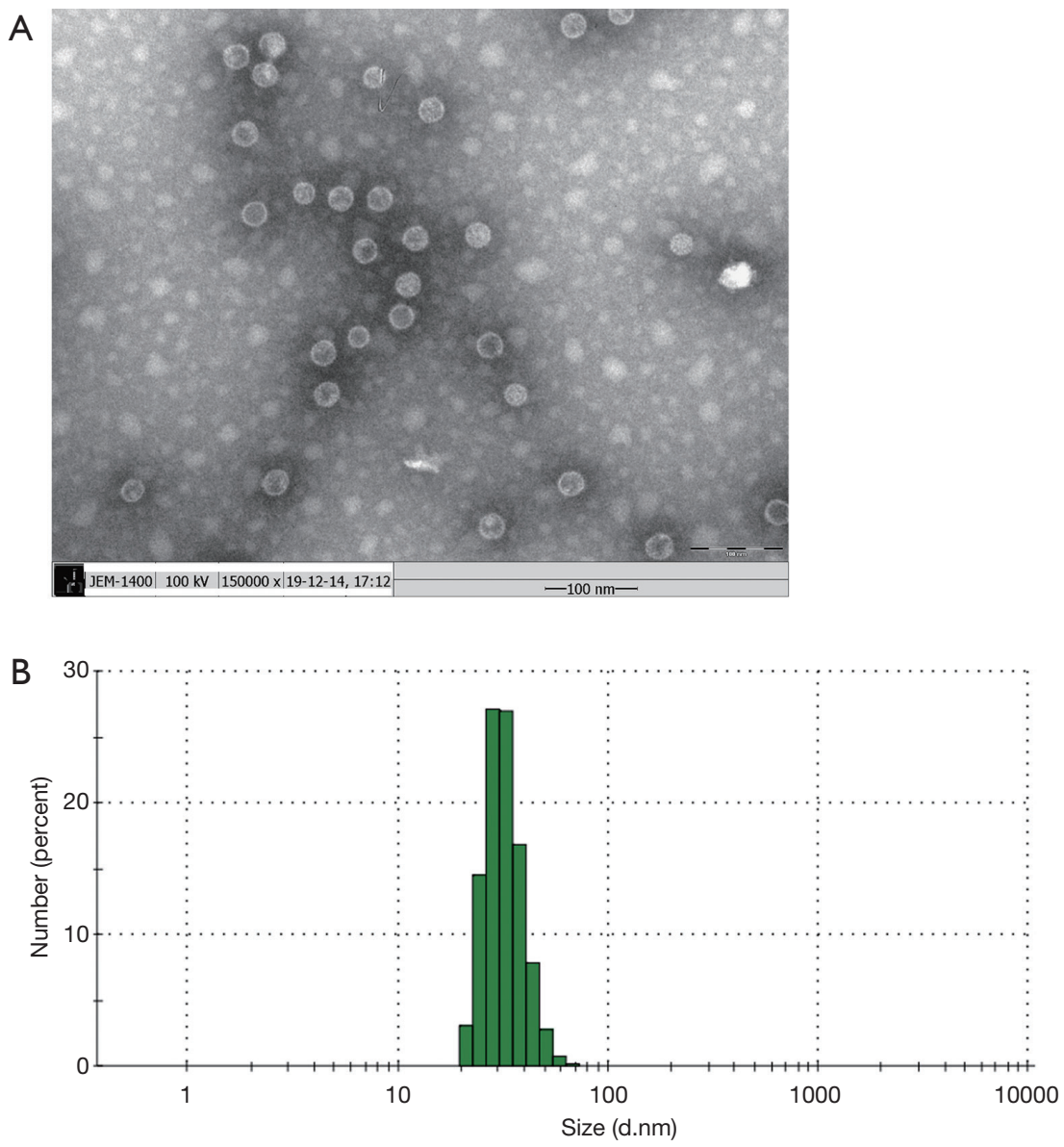

Figure 3 TEM (A), and particle size and potential distribution (B) of the RGD-HBc/ICG VLPs. TEM, transmission electron microscopy; RGD, Arg-Gly-Asp; HBc, hepatitis B virus core protein; VLPs, virus-like particles.

\section{Photothermal transformation test in vitro}

The basis of the application of nanoparticles in the photothermal treatment of tumors is that they have good photothermal conversion performance. Thus, the photothermal conversion effects of ICG and the RGD$\mathrm{HBc} / \mathrm{ICG}$ VLPs under laser irradiation were investigated. As shown in Figure 6, the temperature of the RGD-HBc/ ICG VLPs rose rapidly to $45^{\circ} \mathrm{C}$ after 120 seconds of continuous laser irradiation, and continued to rise with the extension of laser irradiation time. This result suggested that the RGD-HBc VLPs did not affect the photothermal effect of ICG, and even if ICG was contained within the RGD-HB VLPs, it could be converted into photothermal tumor therapy.

\section{Singlet oxygen detection}

Under identical conditions, the RGD-HBc/ICG VLPs could produce more singlet oxygen $\left({ }^{1} \mathrm{O}_{2}\right)$ than ICG (Figure 7A). In addition, we explored the production of ${ }^{1} \mathrm{O}_{2}$ using different concentrations of the RGD-HBc/ICG VLPs under the same conditions. The production of ${ }^{1} \mathrm{O}_{2}$ was concentration dependent (Figure 7B).

\section{Cell uptake experiment}

The DAPI (4',6-diamidino-2-phenylindole) fluorescent dye can bind to DNA strongly. It can be used to dye living cells and fixed cells by penetrating the cell membrane. Under the fluorescence microscope, the nucleus of DAPI-stained cells 


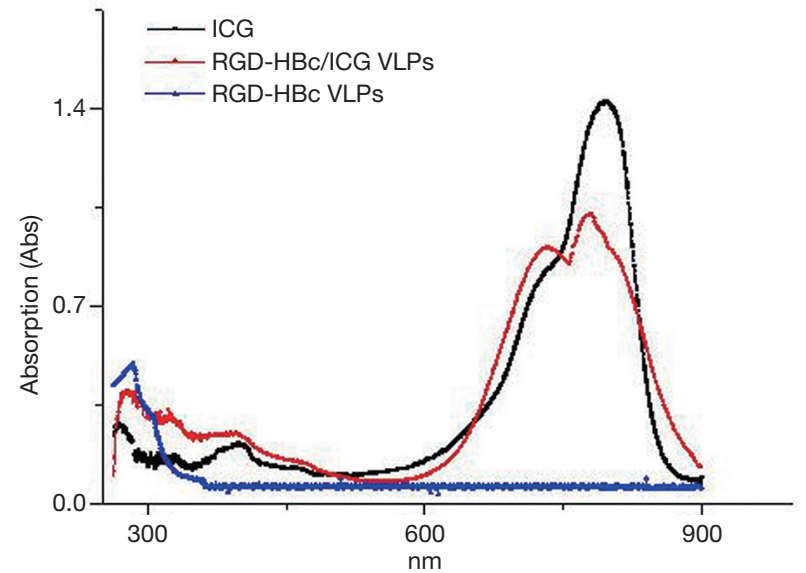

Figure $4 \mathrm{UV}$-vis full-wavelength spectrum. UV, ultraviolet visible; ICG, indocyanine green; RGD, Arg-Gly-Asp; HBc, hepatitis B virus core protein; VLPs, virus-like particles.
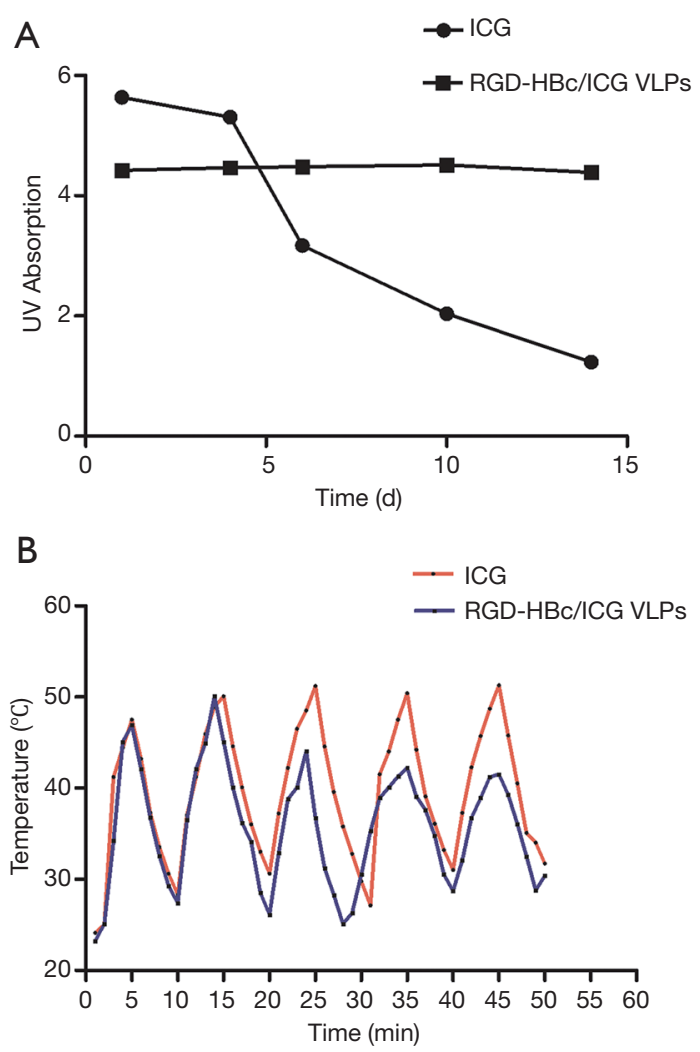

Figure 5 The stability test of the RGD-HBc/ICG VLPs. (A) Time stability; and (B) optical stability (under $808 \mathrm{~nm}$ laser irradiation, $1.5 \mathrm{~W} / \mathrm{cm}^{2}$ ) analyses. PBS, phosphate buffered saline; ICG, indocyanine green; RGD, Arg-Gly-Asp; $\mathrm{HBc}$, hepatitis B virus core protein; VLPs, virus-like particles; d, days..
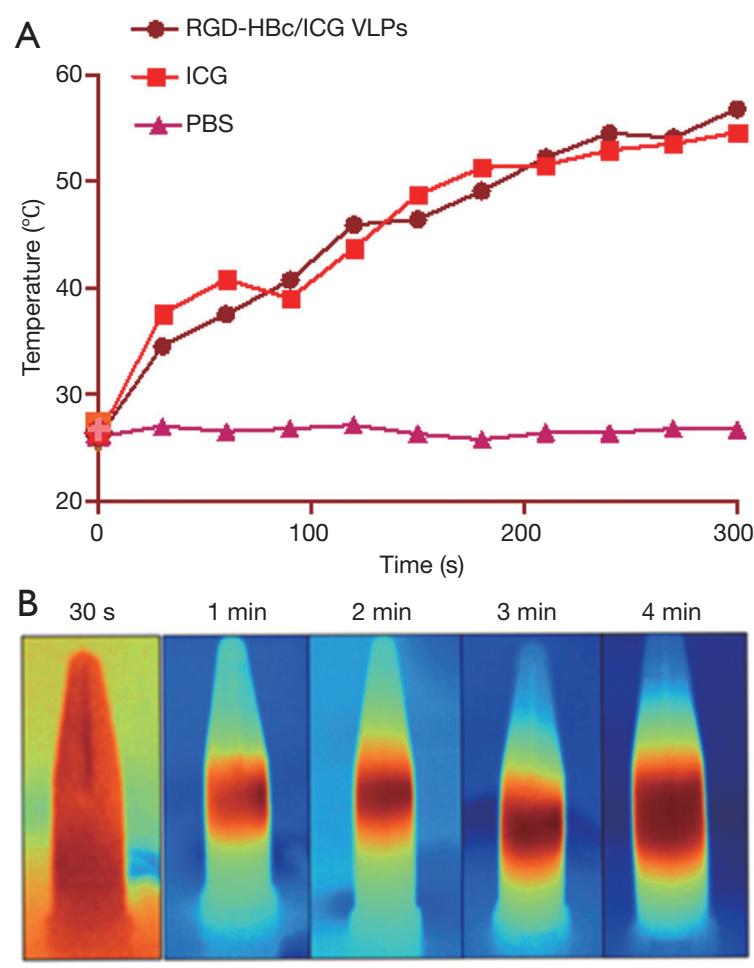

Figure 6 The photothermal conversion property of RGD-HBc/ ICG VLPs in vitro. (A) Temperature changes and (B) infrared thermal imaging at $808 \mathrm{~nm}\left(1 \mathrm{~W} / \mathrm{cm}^{2}, 5 \mathrm{~min}\right)$. RGD, Arg-GlyAsp; $\mathrm{HBc}$, hepatitis B virus core protein; PBS, phosphate buffered saline; ICG, indocyanine green; VLPs, virus-like particles.

are visualized as blue fluorescence, while the near-infrared dye ICG produces red fluorescence after excitation (19). In order to investigate the uptake of the RGD-HBc/ ICG VLPs, 4T1 breast cancer cells were incubated with free ICG or the RGD-HBc/ICG VLPs for 12 hours. Results are shown in Figure 8. Following incubation of the cells with the ICG solution, it was observed that the red fluorescence around the cell nucleus was weak. However, cells incubated for 12 hours with the RGD-HBc/ICG VLPs showed strong red fluorescence signal around the cell nucleus. These results demonstrated that the RGD-HBc/ ICG VLPs have tumor targeting specificity. In addition, the histopathological examination demonstrates that HBc/ICG VLPs have good biological safety.

\section{Light kill experiment}

To explore whether the RGD-HBc/ICG VLPs could 

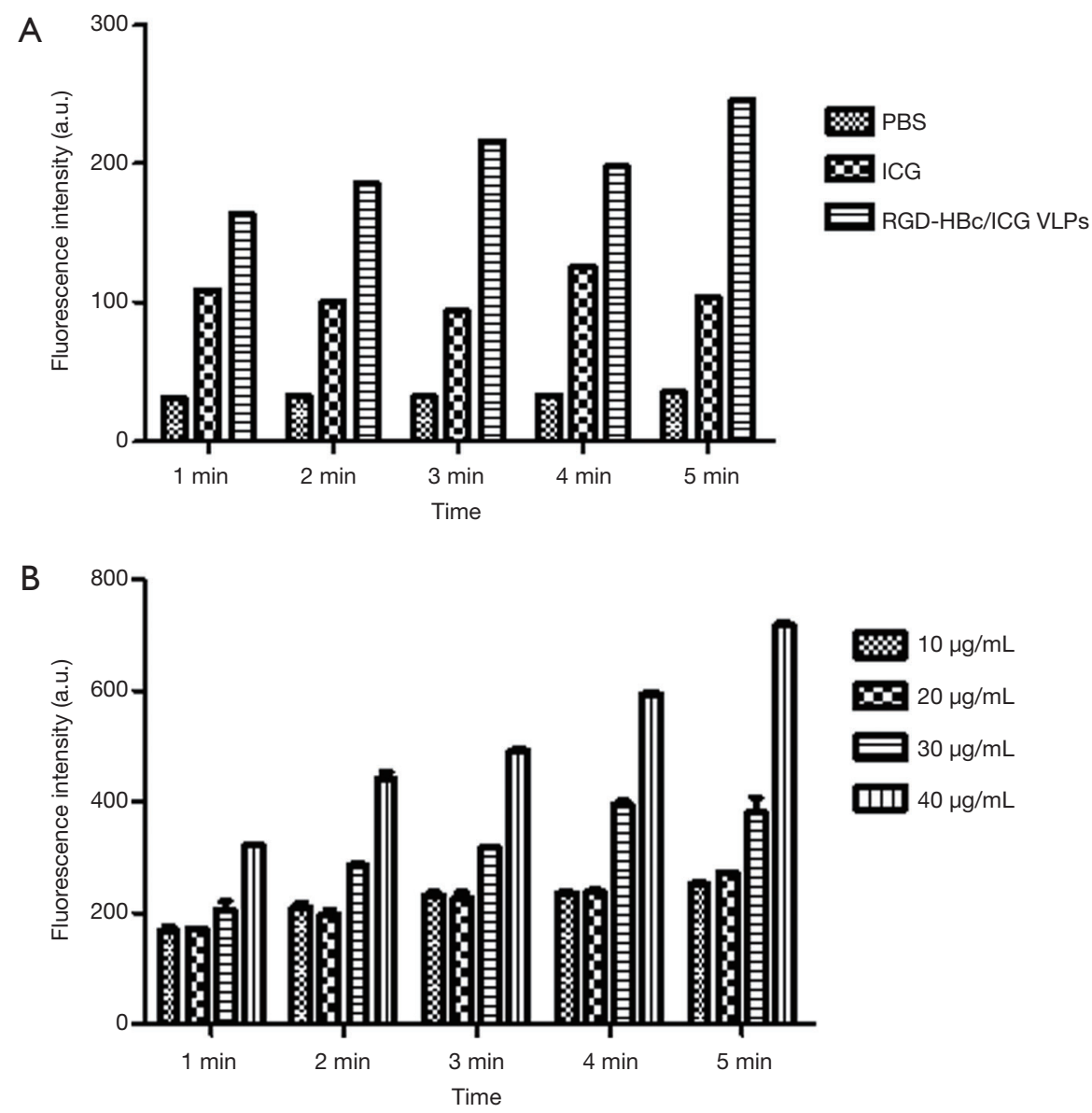

Figure 7 Singlet oxygen detection of RGD-HBc/ICG VLPs. (A) A comparison of ${ }^{1} \mathrm{O}_{2}$ produced by PBS, ICG, and RGD-HBc/ICG VLPs under laser irradiation; (B) a comparison of ${ }^{1} \mathrm{O}_{2}$ produced by different concentrations of RGD-HBc/ICG VLPs under laser irradiation. ${ }^{1} \mathrm{O}_{2}$, singlet oxygen; PBS, phosphate buffered saline; ICG, indocyanine green; RGD, Arg-Gly-Asp; HBc, hepatitis B virus core protein; VLPs, virus-like particles.

continue to play the role of photothermal transformation in cytological experiments, 4T1 cells were incubated with the RGD-HBc/ICG VLPs for 12 hours and then irradiated with an $808 \mathrm{~nm}$ laser $\left(1.5 \mathrm{w} / \mathrm{cm}^{2}, 5 \mathrm{~min}\right)$. The antitumor effects of the RGD-HBc/ICG VLPs were then determined using the Cell Counting Kit-8 (CKK-8) reagent. The results are shown in Figure 9. Following laser irradiation, ICG and the RGD-HBc/ICG VLPs showed better antitumor effect than control, and the effect was greater for the RGD-HBc/ICG VLPs than for free ICG. This may be due to the protective effect of the RGD-HBc VLPs on ICG.

\section{Near-infrared fluorescence in vivo imaging}

To verify the targeting imaging ability of the RGD-
$\mathrm{HBc} / \mathrm{ICG}$ VLPs in 4T1 breast cancer bearing mice, the IVIS lumina III small animal living system (PerkinElmer, Waltham, MA, USA) was used for near-infrared fluorescence imaging. From the images in Figure 10, it can be seen that the RGD-HBc/ICG VLPs reached the tumor site at 2 hours. At 12 hours, the intensity of the fluorescence signal in the tumor site was significantly different from that in the surrounding tissue. The tumor still showed strong fluorescence at 24 hours. However, in the ICG group, no tumor targeting phenomenon was observed at 12 hours. At 24 hours, the fluorescence of the ICG group was almost undetectable, suggesting that the ICG was almost completely metabolized.

After in vivo imaging, the mice were sacrificed, and the excised organs and tumors were examined. The results 

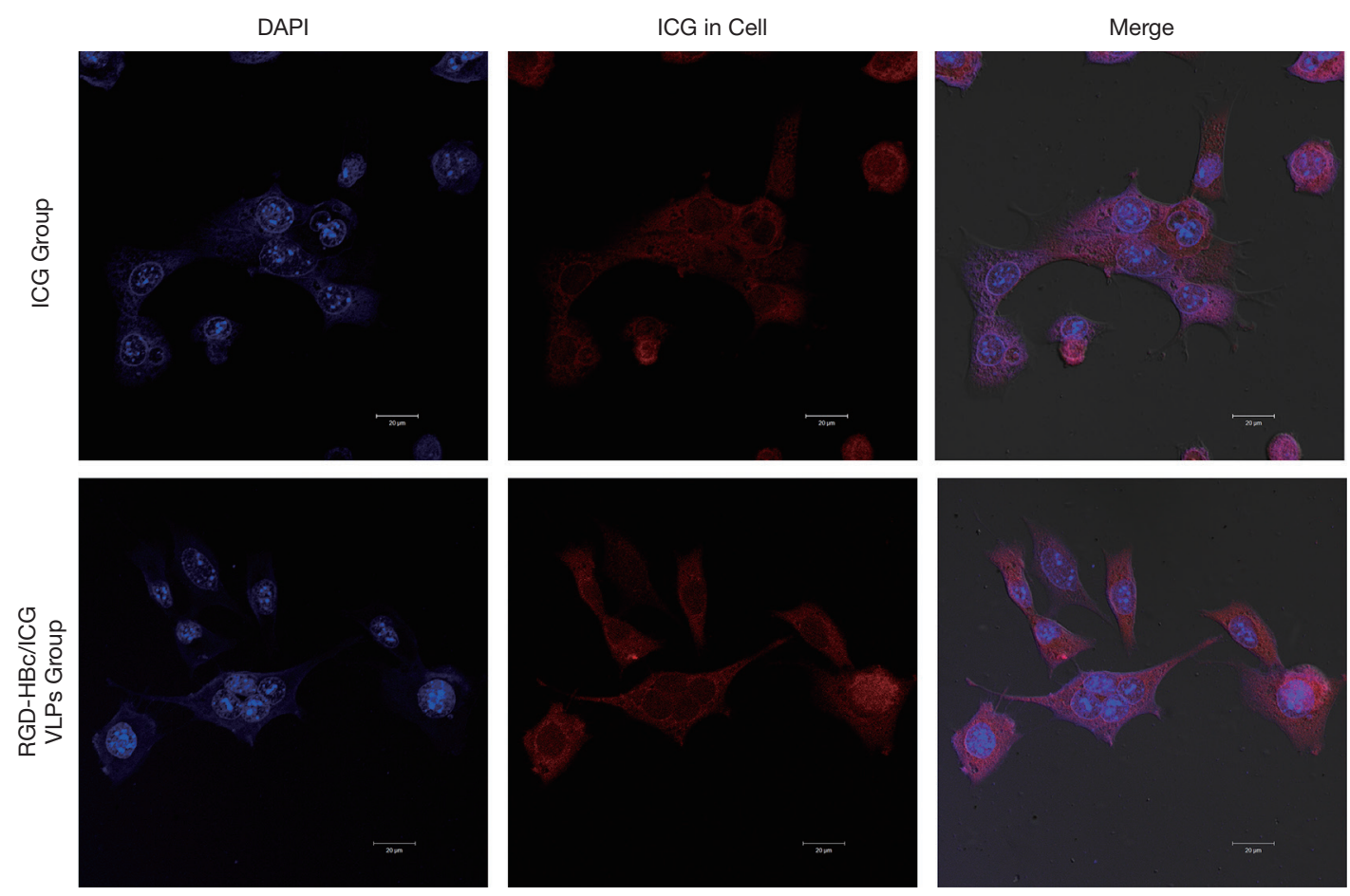

Figure 8 Confocal laser scanning microscope images of 4T1 cells after incubation with ICG and RGD-HBc/ICG for 12 hours. ICG, indocyanine green; RGD, Arg-Gly-Asp; HBc, hepatitis B virus core protein; VLPs, virus-like particles.

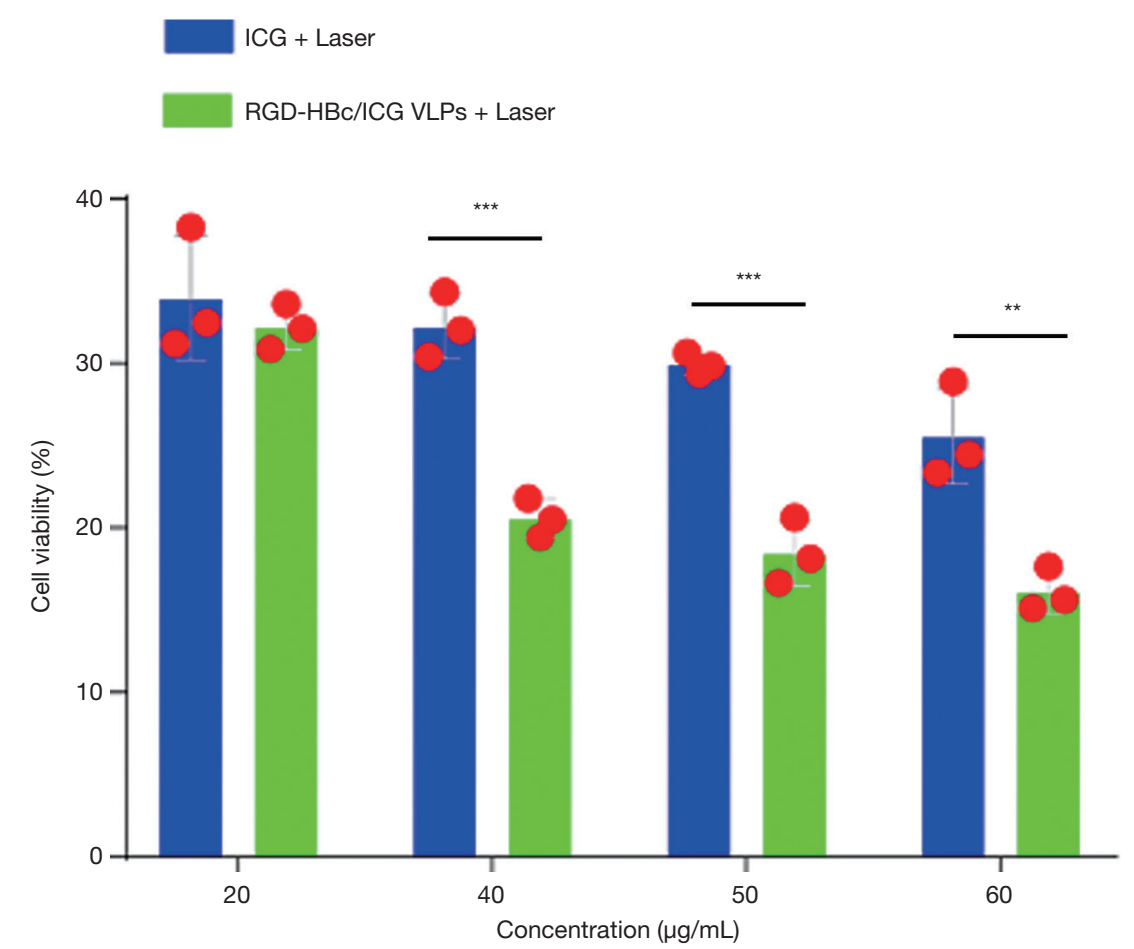

Figure 9 The viability of 4T1 cells treated with ICG and RGD-HBc/ICG VLPs at various concentrations with laser irradiation. ICG, indocyanine green; RGD, Arg-Gly-Asp; HBc, hepatitis B virus core protein; VLPs, virus-like particles. ${ }^{* *}, \mathrm{P}<0.01 ;{ }^{* * *}, \mathrm{P}<0.001$. 
A $2 \mathrm{~h}$ $12 \mathrm{~h}$ $24 \mathrm{~h}$

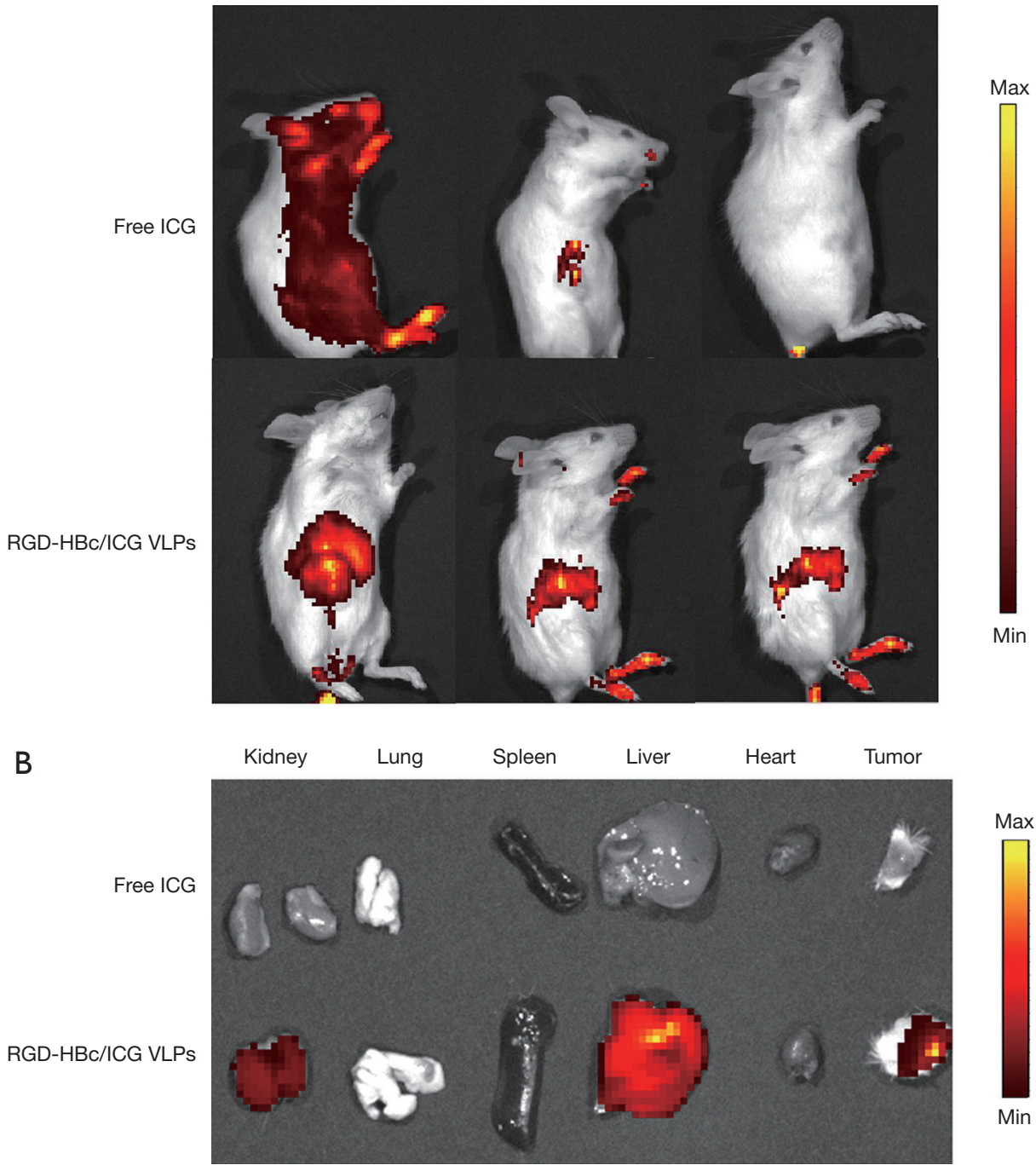

Figure 10 In vivo near-infrared fluorescence imaging of breast cancer mice treated with free ICG or RGD-HBc/ICG VLPs. (A) In vivo fluorescence imaging of 4T1 breast cancer bearing mice treated with free ICG or RGD-HBc/ICG VLPs. (B) The distribution of biological tissue in tumor bearing mice with breast cancer injected with ICG or RGD-HBc/ICG VLPs. ICG, indocyanine green; RGD, Arg-Gly-Asp; $\mathrm{HBc}$, hepatitis B virus core protein; VLPs, virus-like particles.

demonstrated strong signals in the tumors of the RGD$\mathrm{HBc} / \mathrm{ICG}$ VLP group. However, almost no signal was observed in the tumors from the free ICG group.

\section{Conclusions}

The present research examined a specific tumor targeting near-infrared (NIR) nanoparticle by entrapping ICG within the RGD-HBc VLPs. Both in vitro and in vivo imaging were evaluated. The RGD-HBc/ICG VLPs showed excellent optical properties compared to free ICG and exhibited highly selective tumor localization and prolonged circulation time in vivo. In addition, we demonstrated that the RGD-HBc/ICG VLPs were effective at killing in vitro tumor cells mediated by light irradiation. We suggest that the RGD-HBc/ICG VLPs will be a very promising diagnostic and therapeutic integrated nanocarrier.

\section{Acknowledgments}

Funding: This research was funded by the Innovation Scientists and Technicians Troop Construction Projects of 
Henan Province, Grant number 192101510001.

\section{Footnote}

Reporting Checklist: The authors have completed the ARRIVE reporting checklist. Available at http://dx.doi. org/10.21037/atm-20-7478

Data Sharing Statement: Available at http://dx.doi. org/10.21037/atm-20-7478

Conflicts of Interest: All authors have completed the ICMJE uniform disclosure form (available at http://dx.doi. org/10.21037/atm-20-7478). The authors have no conflicts of interest to declare.

Ethical Statement: The authors are accountable for all aspects of the work in ensuring that questions related to the accuracy or integrity of any part of the work are appropriately investigated and resolved. The animal study was reviewed and approved by the Committee on Animal Care and Use of Henan Bioengineering Research Center (Zhengzhou, China). The animal experimental procedures were in accordance with the guidelines approved by the Committee on Animal Care and Use of Henan Bioengineering Research Center (Zhengzhou, China).

Open Access Statement: This is an Open Access article distributed in accordance with the Creative Commons Attribution-NonCommercial-NoDerivs 4.0 International License (CC BY-NC-ND 4.0), which permits the noncommercial replication and distribution of the article with the strict proviso that no changes or edits are made and the original work is properly cited (including links to both the formal publication through the relevant DOI and the license). See: https://creativecommons.org/licenses/by-nc-nd/4.0/.

\section{References}

1. Chaudhary Z, Khan GM, Abeer MM, et al. Efficient photoacoustic imaging using indocyanine green (ICG) loaded functionalized mesoporous silica nanoparticles. Biomater Sci 2019;7:5002-15.

2. Li W, Yang J, Luo L, et al. Targeting photodynamic and photothermal therapy to the endoplasmic reticulum enhances immunogenic cancer cell death. Nat Commun 2019;10:3349.

3. Shi J, Kantoff PW, Wooster R, Farokhzad OC. Cancer nanomedicine: progress, challenges and opportunities. Nat Rev Cancer 2017;17:20-37.

4. Chen XJ, Zhang XQ, Liu Q, et al. Nanotechnology: a promising method for oral cancer detection and diagnosis. J Nanobiotechnology 2018;16:52.

5. Crowther RA, Kiselev NA, Böttcher B, et al. Threedimensional structure of hepatitis $B$ virus core particles determined by electron cryomicroscopy Cell 1994;77:943-50.

6. Pumpens P, Grens E. The true story and advantages of the famous Hepatitis B virus core particles: Outlook 2016. Mol Biol (Mosk) 2016;50:558-76.

7. Strods A, Ose V, Bogans J, et al. Preparation by alkaline treatment and detailed characterisation of empty hepatitis $\mathrm{B}$ virus core particles for vaccine and gene therapy applications. Sci Rep 2015;5:11639.

8. Clarke BE, Newton SE, Carroll AR, et al. Improved immunogenicity of a peptide epitope after fusion to hepatitis B core protein. Nature 1987;330:381-4.

9. Schödel F, Moriarty AM, Peterson DL, et al. The position of heterologous epitopes inserted in hepatitis B virus core particles determines their immunogenicity. J Virol 1992;66:106-14.

10. Duan XY, Wang JS, Guo YM, et al. Antitumor effect of EGFRvIII/HBcAg recomibinant vaccine on murine transplanted tumor. Xi Bao Yu Fen Zi Mian Yi Xue Za Zhi 2007;23:600-2.

11. Krieg AM. CpG motifs in bacterial DNA and their immune effects. Annu Rev Immunol 2002;20:709-60.

12. Song S, Wang Y, Zhang Y, et al. Augmented induction of CD8+ cytotoxic T-cell response and antitumor effect by DCs pulsed with virus-like particles packaging with CpG. Cancer Lett 2007;256:90-100.

13. Shan $\mathrm{W}$, Zhang $\mathrm{D}, \mathrm{Wu} \mathrm{Y}$, et al. Modularized peptides modified $\mathrm{HBc}$ virus-like particles for encapsulation and tumor-targeted delivery of doxorubicin. Nanomedicine 2018;14:725-34.

14. Yamada T, Iwasaki $Y$, Tada H, et al. Nanoparticles for the delivery of genes and drugs to human hepatocytes. Nat Biotechnol 2003;21:885-90.

15. Shan W, Zhang D, Wu Y, et al. Modularized peptides modified $\mathrm{HBc}$ virus-like particles for encapsulation and tumor-targeted delivery of doxorubicin. Nanomedicine 2018;14:725-34.

16. Li CH, Yang J, Ren J, et al., Preparation of tuftsin functionalized HBc VLPs nanocarrier. Chin J Biochem Mol Biol 2020;36:804-10.

17. Yoon HJ, Lee HS, Lim JY, Park JH. Liposomal 
indocyanine green for enhanced photothermal therapy. ACS Appl Mater Interfaces 2017;9:5683-91.

18. Hwang J, Jin JO. Attachable hydrogel containing indocyanine green for selective photothermal therapy against melanoma. Biomolecules 2020;10:1124.

Cite this article as: Wang $\mathrm{Y}$, Zhang $\mathrm{Y}$, Yu Y, Ren L, Wang J, Cheng L, Jiang D, Guo X, Teng T, Luo X, Lv S, Wang X, Wang H, Shi X, Zhang H, Bi S. Preparation and preliminary evaluation of hepatitis $\mathrm{B}$ core antigen virus like nanoparticles loaded with indocyanine green. Ann Transl Med 2020;8(24):1661. doi: 10.21037/atm-20-7478
19. Williamson DH, Fennell DJ. The use of fluorescent DNA-binding agent for detecting and separating yeast mitochondrial DNA. Methods Cell Biol 1975; 12:335-51.

(English Language Editor: J. Teoh) 